\title{
Multi-step Identification Algorithm for the Multi-sensor Target Tracking System
}

\author{
Li Heng ${ }^{1+}$, Chen Jia-qi ${ }^{1}$, Wang Hao ${ }^{1}$ and Ni Jin-ying ${ }^{1}$ \\ ${ }^{1}$ School of Computer and Information Engineering, Fuyang Normal University, Fuyang, Anhui, 236037,
}

China

\begin{abstract}
For a multi-sensor target tracking system, when the measurement noise of all the sensors are uncertain, this paper presents a kind of algorithm to estimate the parameters and noise statistics information. This algorithm consists of 2 steps, the first step is to use the recursive least squares method to get the estimations of the model parameters, the second step is to use the correlation function method to get the estimations of the noise statistics. The convergence of this algorithm is proved by mathematical method. An example is given to show the effectiveness of this algorithm.
\end{abstract}

Keywords: multi-sensor, target tracking system, measurement noise, recursive least squares method, correlation function method

\section{Introduction}

Multi-sensor information fusion theory is also called multi-source information fusion. In order to obtain the optimal estimation of the state and other related information, each source's information is combined by certain algorithm[1]. Multi-sensor information fusion theory has been widely used in military command, control, communication and target tracking systems. In the target tracking problem, the main question is to get the optimal estimation of the position, velocity and acceleration of the target. In general, the state space equation can be used to describe the changing law of the state of the target, and the observation equation is used to describe the relationship between the observed results and the actual state[2-5]. Filtering the signals from the observed results of noise pollution is key problem of the estimation. In the past target tracking references like [9-16], in general, the estimations of the measurement noise statistics are not considered. But in the real applications, when the target is in a complicated environment such as the extreme temperature and humidity, the measurement noise statistics are always different in the whole filtering process[17-19]. So it is necessary to estimate the statistical information of the measurement noise of every sensors before the filtering process. For the multi-sensor target tracking system with unknown noise statistics, this paper presents an algorithm to get the estimations of the measurement noise. This algorithm consists of the recursive least square method and correlation method. We prove the convergence of this algorithm by mathematical method. An example is given to show the effectiveness of this algorithm.

\section{Problem Formulation}

We assume that the state equation could be described as the AR model form, it has

$$
A\left(q^{-1}\right) s(t)=w(t)
$$

The observation equation is

$$
y_{i}(t)=s(t)+v_{i}(t)+u(t), i=1, \cdots, L
$$

\footnotetext{
+ Corresponding author. Tel.: +(0086 13345582728); fax: +(0086 05583222345).

E-mail address: (shenzhou5hao0519@163.com).
} 
where $\mathrm{t}$ is the sampling time, and $s(t) \in R^{n}$ is the AR model signal, $L$ is the number of the sensors, $v_{i}(t) \in R^{m i}$ is the measurement noise, $\mathrm{u}(t)$ is the control law, $A\left(q^{-1}\right)$ is a polynomial with the form

$$
A\left(q^{-1}\right)=1+a_{1} q^{-1}+\ldots+a_{n} q^{-n}
$$

$q^{-1}$ is the delay factor, $q^{-1} x(t+1)=x(t)$.

Assumption 1. $y_{i}(t)(i=1, \cdots, L)$ is bounded.

Assumption 2. The polynomial $A\left(q^{-1}\right)$ is stable.

Assumption 3. The parameters of $A\left(q^{-1}\right)$ is unknown, the measurement noise variance of every single sensor is unknown.

With the assumption1-3, the question is for a target tracking system, how to get the unbiased estimates of $A\left(q^{-1}\right)$ and the measurement noise variance.

\section{Algorithm}

\subsection{The First Step}

Substituting (1) into (2), we can get

$$
A\left(q^{-1}\right) y_{i}(t)=w(t)+u(t)+A\left(q^{-1}\right) v_{i}(t)
$$

We can define

$$
\begin{gathered}
z_{i}(t)=A\left(q^{-1}\right) y_{i}(t)-u(t) \\
\theta=\left(a_{1}, \ldots, a_{n}\right)^{T} \\
\varphi_{i}^{T}(t)=\left(-z_{i}(t-1), \ldots,-z_{i}(t-n)\right)^{T} \\
e_{i}(t)=w(t)+A\left(q^{-1}\right) v_{i}(t)
\end{gathered}
$$

(4) can be described by the least squares form

$$
z_{i}(t)=\varphi_{i}^{T}(t) \theta+e_{i}(t)
$$

We use the recursive least squares method, it has the relationship

$$
\begin{gathered}
\hat{\theta}_{i}(t)=\hat{\theta}_{i}(t-1)+\frac{P_{i}(t-1) \hat{\varphi}_{i}(t)\left[z_{i}(t)-\varphi_{i}^{\mathrm{T}}(t) \hat{\theta}_{i}(t-1)\right]}{1+\varphi_{i}^{\mathrm{T}}(t) P_{i}(t-1) \hat{\varphi}_{i}(t)} \\
P_{i}(t)=P_{i}(t-1)-\frac{P_{i}(t-1) \hat{\varphi}_{i}(t) \varphi_{i}^{\mathrm{T}}(t) P_{i}(t-1)}{1+\varphi_{i}^{\mathrm{T}}(t) P_{i}(t-1) \hat{\varphi}_{i}(t)}
\end{gathered}
$$

We use the method of taking the average of all the estimates of the sensors, so it has the relationship

$$
\hat{A}_{f}\left(q^{-1}\right)=\frac{1}{L} \sum_{i=1}^{L} \hat{A}_{i}\left(q^{-1}\right)
$$

In (12), the subscript $\mathrm{f}$ means the fusion results by multiple sensors.

\subsection{The Second Step}

Due to the assumption 1, we know $y_{i}(t)$ and $z_{i}(t)$ both have the ergodic property, according to the definition of the self-correlation function we have

$$
R_{z i i}(k)=\mathrm{E}\left[z_{i}(t) z_{i}(t-k)\right]
$$

According to (5), $R_{z i i}(k), k=0, \ldots, n_{a}$ could be obtained

$$
\begin{gathered}
R_{z i i}(0)=\left(1+a_{1}^{2}+\ldots+a_{n}^{2}\right) R_{y i i}(0)+R_{u}(0) \\
R_{z i i}(k)=\sum^{n_{a}} a_{j} a_{j+k} R_{y i i}(k)+R_{u}(k)
\end{gathered}
$$

$u(t)$ is control law which is described as the form

$$
u(t)=\mathrm{B}\left(q^{-1}\right) \mathrm{m}(\mathrm{t})=\left(1+b_{1} q^{-1}+\ldots+b_{n_{b}} q^{-n_{b}}\right) \mathrm{m}(\mathrm{t})
$$

The order of $\mathrm{B}\left(q^{-1}\right)$ is $n_{b}$ which is less than $n_{a}$, we set $u(t)$ as a linear time series to deal with the changes of $y_{i}(t)$. The functions(14) and (15) constitute a set of linear equations.

According to (4), we have the functions 


$$
\begin{gathered}
R_{z i i}(0)=\sigma_{w}^{2}+\sigma_{v i}^{2} \\
R_{z i i}(k)=\sigma_{v i}^{2} \sum^{n} a_{u} a_{u-k}, \quad k=1, \ldots, n_{a}
\end{gathered}
$$

The estimations of $\sigma_{w}^{2}$ and $\sigma_{v i}^{2}$ could be solved in $(17)$ and (18), we can remark them as $\hat{\sigma}_{w}^{2}$ and $\hat{\sigma}_{v i}^{2}$. $\hat{\sigma}_{v i}^{2}$ is the estimation of the statistics of the measurement noise.

Theorem 1 Optimal Kalman filter For the multiple sensors system with the assumptions 1-3, the noise statistics of every single sensors estimated by the algorithm have convergence to the real values.

Proof: From(5)-(7), according to the convergence of the recursive least squares method, we can get

$$
\hat{\theta}_{i}(t) \rightarrow \theta, t \rightarrow \infty, \text { w.p. } 1
$$

w.p. 1 is short for "with probability 1 ". According to the consistency of the elementary mathematics, the fusion estimates also have the convergence to the real values, so we have

$$
\hat{\theta}_{f}(t) \rightarrow \theta, t \rightarrow \infty \text {,w.p. } 1
$$

In (20), $\theta$ can be replaced by $A\left(q^{-1}\right)$, so we have

$$
\hat{A}_{f}\left(q^{-1}\right) \rightarrow A\left(q^{-1}\right), t \rightarrow \infty, \text { w.p. } 1
$$

According to the ergodic property of the correlation function, and $u(t)$ is a linear time series, from (14) and (15), we can get

$$
\hat{\sigma}_{v i}^{2} \rightarrow \sigma_{v i}^{2}, t \rightarrow \infty \text {,w.p. } 1
$$

So we can get the estimates of measurement noise have convergence to their real values.

\section{Example}

We consider about a 3 -sensors target tracking system which has the forms as (1) and(2)

$$
\begin{gathered}
A\left(q^{-1}\right) s(t)=w(t) \\
y_{i}(t)=s(t)+v_{i}(t)+u(t), i=1, \cdots, L
\end{gathered}
$$

We suppose the order of the polynomial $A\left(q^{-1}\right), n_{a}=2$, the parameters $a_{1}=0.9, a_{2}=0.66$, $\sigma_{v 1}^{2}=0.1, \sigma_{v 2}^{2}=0.2, \sigma_{v 3}^{2}=0.3$. We use matlab to simulate this example, the simulation results show the effectiveness of the algorithm. In these figures, the straight lines say the real value, the solid-line curves say the fusion value, the imaginary curves say the local value. We can see that in the figure $1-4$, the estimations all have convergence to the real values.

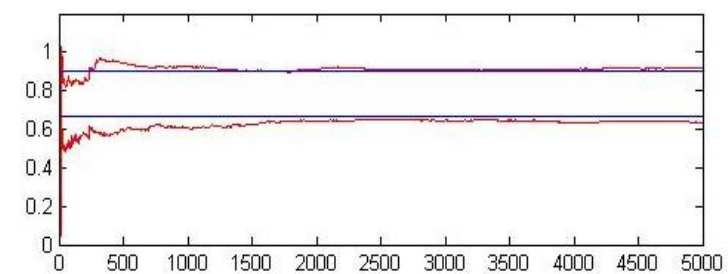

Fig. 1: The curve of the estimates of $a_{1}$ and $a_{2}$.

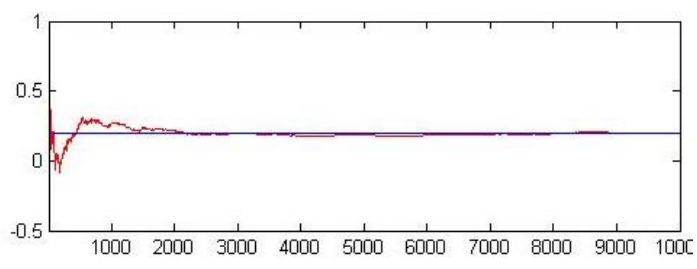

Fig. 3: The curve of the estimate of $\sigma_{v 2}^{2}$.

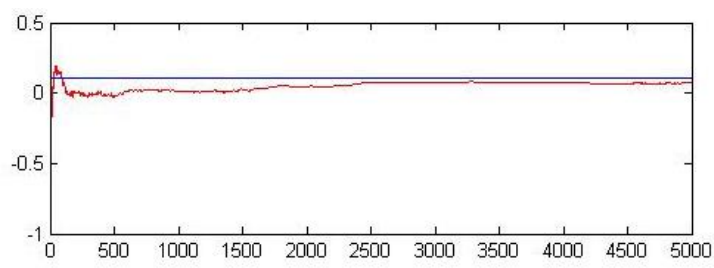

Fig. 2: The curve of the estimate of $\sigma_{v 1}^{2}$.

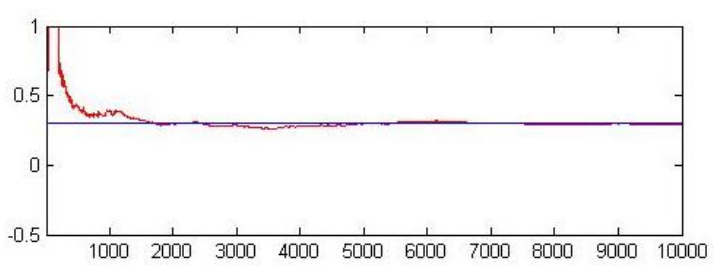

Fig. 4: The curve of the estimate of $\sigma_{v 3}^{2}$.

\section{Conclusion}

For the multi-sensor target tracking system, when the statistics information of the measurement noise are uncertain, in order to improve the accuracy of the measurement noise statistics, this paper presents a kind 
of algorithm. We prove the convergence of this algorithm by mathematical way. An example is given to simulate some figures shows the effectiveness of this algorithm, in these figures, we find the estimations all have good convergence to the real values, which shows the good effectiveness of this algorithm

\section{Acknowledgment}

This paper is supported by National Undergraduate Innovative Training Program(No.201710371007); National Science Foundation of China(No.61401101; 61673117); Fuyang Normal University Science Research Project (2015FSKJ11; 2016FSKJ16); Anhui Education Project (No. KJ2016A561).

\section{References}

[1] Zili Deng. Information Fusion Estimation theory and applications. Science Press, 2012.

[2] Gao Y, Xa H Q, Deng Z L., "Multi-stage information fusion identification method for multisensor ARMA signals with white measurement noises," 2010 8th IEEE International Conference on Control and Automation,vol.10,no.2,pp.1115-1119,2010.

[3] J. F. Liu, Z. L. Deng., "An information fusion identification method for multisensor autoregressive moving average signals with white measurement noise and sensor bias", Sensor Letters, vol.9, no,1, pp.1443-1447,2011.

[4] Ran C J, Deng Z L., "Self-tuning weighted measurement fusion Kalman filter and its convergence. Journal of Control Theory and Applications", vol.8, no.4, pp.435-440,2010.

[5] NEAL.A.CARLSON, "Federated Square Root Filter for Decentralized Parallel Processes", IEEE Transactions on Aerospace and Electronic", vol.8, no.4, pp.435-440,2010.

[6] Ran C J, Deng Z L. Self-tuning weighted measurement fusion Kalman filter and its convergence. Journal of Control Theory and Applications,vol.8,no.4,pp.435-440,2010. Zili Deng. Self-tuning filtering theory and applications. Harbin Institute of Technology Press, 2003.

[7] Z. L. Deng, "Information Fusion Estimation Theory and its Application”,Science Press,Beijing,China,2012.

[8] Shu-Li Sun, Z.L.Deng. "Multi-sensor Optimal Information Fusion Kalman Filter", Automatica, vol. 50, no. 1, pp. 1017-1023, 2004.

[9] DENG Zi-li,LI Chun-Bo,"Self-tuning Information Fusion Kalman Predictor Weighted by Diagonal Matrices and Its Convergence Analysis", ACTA Automatica, SINICA,vol.33,no.2,pp.156-163, 2007.

[10] Zili Deng, Yuan Gao, Chunbo Li, Gang Hao, "Self-tuning decoupled information fusion wiener state component filters and their convergence", Automatica,vol.44,no.3,pp.685-695,2008.

[11] Gao Y, Ran C J, Sun X J, et al. Optimal and self-tuning weighted measurement fusion Kalman filters and their asymptotic global optimality. International Journal of Adaptive Control and Signal Processing, vol.24, no.1, pp.982-1004, 2010.

[12] K. J. Astrom, B. Wittenmark, “On self tuning regulators”, Automatica, 1973, 9:185-199.

[13] Ran C J, Deng Z L. Self-tuning weighted measurement fusion Kalman filter and its convergence. Journal of Control Theory and Applications, vol.8, no.4, pp.435-440, 2010.

[14] Deng Z L, "Self-tuning $\alpha-\beta-\gamma$ Tracking Filter", Control and Decision, vol.6, no.5, pp.384-387, 1992.

[15] Deng Z L, "Self-tuning $\alpha-\beta$ Tracking Filter", ACTA Automatica SINICA, vol.18, no.6, pp.720-723, 1993.

[16] P. J. Dua, S. C. Liu, J. S. Xia and Y. D. Zhao, "Information fusion techniques for change detection from multitemporal remote sensing images", Information Fusion, vol. 14, no. 1,pp. 19-27,2013.

[17] Kishore Mehrotra, Pravas R.Mahapatra, “A Jerk Model for Tracking Highly Maneuvering Target”, IEEE Transactions on Aerospace and Electronics Systems,vol.33, no.4, pp.1094-1105, 1997.

[18] Shu-Li Sun, Z.L.Deng. “Multi-sensor Optimal Information Fusion Kalman Filter”,Automatica,vol.50,no.1,pp.10171023,2004 .

[19]DENG Zi-li,LI Chun-Bo,"Self-tuning Information Fusion Kalman Predictor Weighted by Diagonal Matrices and Its Convergence Analysis", ACTA Automatica SINICA,vol.33,no.2,pp.156-163,2007. 